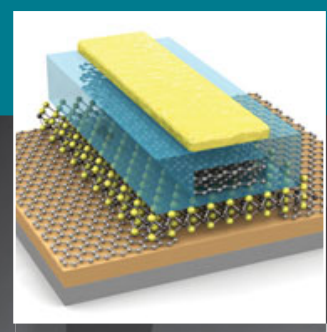

\title{
Electronic properties of transition-metal dichalcogenides
}

\author{
Agnieszka Kuc, Thomas Heine, and Andras Kis
}

\begin{abstract}
Graphene is not the only prominent example of two-dimensional (2D) materials. Due to their interesting combination of high mechanical strength and optical transparency, direct bandgap and atomic scale thickness transition-metal dichalcogenides (TMDCs) are an example of other materials that are now vying for the attention of the materials research community. In this article, the current state of quantum-theoretical calculations of the electronic and mechanical properties of semiconducting TMDC materials are presented. In particular, the intriguing interplay between external parameters (electric field, strain) and band structure, as well as the basic properties of heterostructures formed by vertical stacking of different 2D TMDCs are reviewed. Electrical measurements of $\mathrm{MoS}_{2}, \mathrm{WS}_{2}$, and $\mathrm{WSe}_{2}$ and their heterostructures, starting from simple field-effect transistors to more demanding logic circuits, high-frequency transistors, and memory devices, are also presented.
\end{abstract}

\section{Introduction}

After quantum dots, nanowires, and nanotubes, two-dimensional (2D) materials in the form of sheets with atomic-scale thickness represent the most recent nanoscale material family under intense study. These materials appear in their bulk form as stacks of layers held together via van der Waals interaction in crystals. Single layers with atomic-scale thickness can be extracted from such crystals. The best-known example is graphite, composed of individual graphene layers. Twodimensional materials are attractive for use in electronic devices because, compared to nanowires and nanotubes, it is easier to fabricate complex structures from them. Their atomicscale thickness makes it possible to tune their properties using external electric fields, while control over the number of layers in mesoscopic structures gives an additional way to modify their electronic and optical properties. ${ }^{1,2}$

The first layered material to be thinned to a single monolayer (ML) was graphite, where the ML is referred to as graphene. ${ }^{3}$ It continues to be widely studied because of its rich physics and high mobility. ${ }^{4}$ However, pristine graphene does not have a bandgap, which is required for many applications in electronics and optoelectronics. Bandgaps can be engineered in graphene, but this increases complexity either reduces mobilities to the level of strained silicon films ( $100 \mathrm{~cm}^{2} / \mathrm{Vs}$ at room temperature) or requires voltages on the order of $100 \mathrm{~V}^{5,6}$
One of the key advances that brought attention to 2D semiconductors was the demonstration of a field-effect transistor (FET) with a high ON/OFF ratio based on a single layer of $\mathrm{MoS}_{2},{ }^{7}$ a semiconducting material from the transition-metal dichalcogenide (TMDC) family. This was the first demonstration of a high-quality device based on a 2D material other than graphene.

$\mathrm{MoS}_{2}$ (Figure 1) is a member of a large family of materials known as TMDCs (please see the Introduction article in this issue of MRS Bulletin). They have the common chemical formula $\mathrm{MX}_{2}$, where $\mathrm{M}$ stands for a transition metal $(\mathrm{M}=\mathrm{Mo}, \mathrm{W}$, $\mathrm{Nb}, \mathrm{Ta}, \mathrm{Ti}, \mathrm{Re}$ ) and $\mathrm{X}$ for $\mathrm{Se}, \mathrm{S}$, or Te. Bulk TMDC crystals are formed by vertical stacking of $2 \mathrm{D}$ layers that are $\sim 6.5 \AA$ thick. Depending on the chemical composition, they can have different electrical properties ranging from semiconducting to superconducting. Single layers can be extracted using the micromechanical cleavage technique, ${ }^{9,10}$ commonly used for the production of graphene or liquid phase exfoliation, a mild solvent-based exfoliation technique. ${ }^{11}$ Large-area $\mathrm{MoS}_{2}$ can be grown using chemical-vapor-deposition-like growth techniques. ${ }^{12,13}$ The lack of dangling bonds also makes it possible to re-stack different $2 \mathrm{D}$ materials in the vertical direction and produce heterostructures ${ }^{14}$ without the requirement of lattice matching.

Around 60 TMDC materials were known in the late 1960s, with around 40 of them having a layered structure ${ }^{15}$ (Table I). 


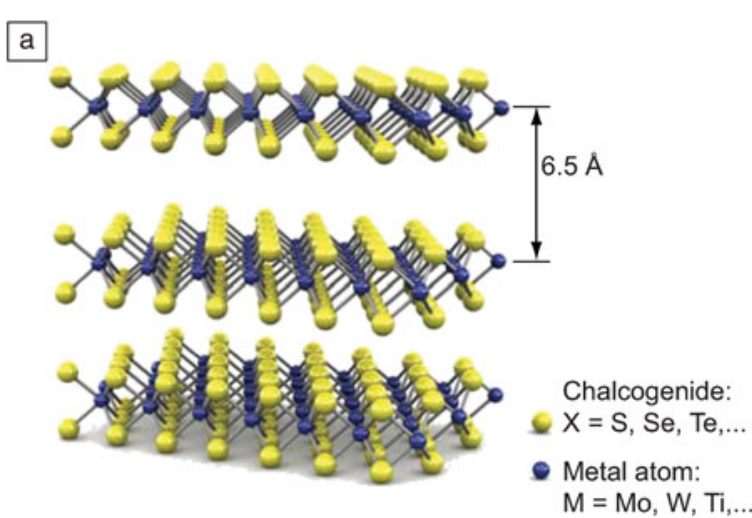

b
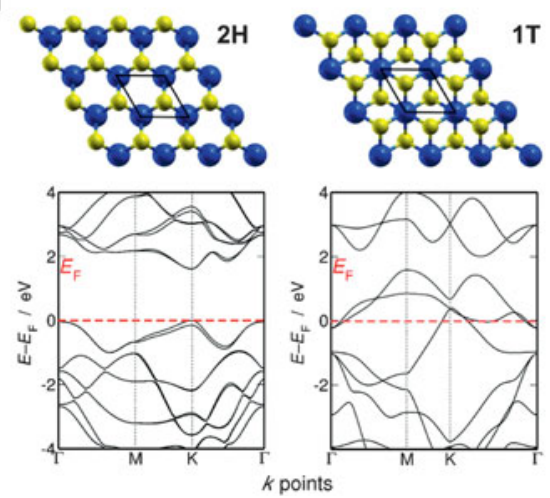

Figure 1. (a) Three-dimensional representation of the structure of transition-metal dichalcogenides (TMDCs) with the common formula $\mathrm{MX}_{2}$. Single layers can be extracted and used as the starting point for fundamental research and device applications. (b) Structure (top) and band structure (bottom) of monolayer $\mathrm{MoS}_{2}$ in $2 \mathrm{H}$, trigonal prismatic, (left) and 1T, octahedral, (right) ${ }^{8}$ symmetry. The Fermi level $\left(E_{\mathrm{F}}\right)$ is indicated by the red dashed line. Courtesy of Nourdine Zibouche (Jacobs University Bremen). Note: $E$, energy.

First reports of ultrathin (10 nm thick) $\mathrm{MoS}_{2}$ date back to $1963,{ }^{16}$ while the production of single-layer $\mathrm{MoS}_{2}$ in suspension was first reported in 1986. ${ }^{17}$ The rapid growth of graphene-related research and the development of new techniques for working with ultrathin layered materials opened new perspectives for the study of 2D TMDC layers. The indication that TMDC MLs are fundamentally different from thicker bulk material came with the realization that the bandgap of $\mathrm{MoS}_{2}$ changes from the bulk value of $1.2 \mathrm{eV}^{18}$ to $1.8 \mathrm{eV}^{19,20}$ as the thickness is decreased down to a ML. This is due to quantum-mechanical confinement in the vertical direction ${ }^{21}$ and the resulting change in hybridization in orbitals related to $\mathrm{M}$ and $\mathrm{X}$ atoms. Moreover, unlike the bulk material, singlelayer $\mathrm{MoS}_{2}$ is a direct gap semiconductor. This seems to be a general feature shared by many other semiconducting TMDCs $\left(\mathrm{MoSe}_{2}, \mathrm{WS}_{2}, \mathrm{WSe}_{2}, \mathrm{MoTe}_{2}\right)$ that are expected to show indirect to direct bandgap transitions in the limit of a single layer, spanning the 1.1-1.9 eV bandgap energy range. ${ }^{21-25}$

Even though a large number of layered TMDCs are known, only a handful of them are widely available in the form of a synthetic material. Most of the recent research efforts are concentrated on semiconducting TMDCs due to their potential for applications in electronics, with $\mathrm{MoS}_{2}$ being the most studied example due to the fact that it is also available in the form of a naturally occurring mineral, molybdenite. ${ }^{26}$

\section{Modeling the electronic structure of TMDCs}

Important contributions to the development of the field of 2D crystals have come from theory. While theory offers an explanation of many fundamental experimental effects, many phenomena have been predicted by computer simulations. In the following, some of these examples are compiled, with a focus on our own contributions. It is important to note that calculations on 2D materials may require different approaches and computational details, so the interested reader is referred to our recent tutorial reviews of the topic. ${ }^{1,36}$

Theoretical modeling offers fast screening of many 2D crystals. We have compiled the structural and electronic properties of a large selection of $2 \mathrm{D}$ crystals, thus providing a database of bandgaps, effective electron and hole masses, lattice parameters, and band structures for further investigation by experiment and theory. ${ }^{37}$ Materials with spectacular properties invite further investigation; for example, in the case of $\mathrm{PdS}_{2}$, a Group 10 TMDC with $1 \mathrm{~T}$ symmetry is semiconducting as a ML, but becomes semimetallic as a bilayer (BL).$^{38}$

When Group 6 semiconducting $-\mathrm{MX}_{2}$ materials with the $2 \mathrm{H}$ (trigonal prismatic) structure type (Figure $1 \mathrm{~b}$ ) are confined from the bulk to the ML, their crystal symmetry lowers, and their electronic structure changes significantly. An important example of this quantum confinement is the observed enhanced photoluminescence signal from a $\mathrm{MoS}_{2} \mathrm{ML}$, which has been rationalized by band structure calculations. ${ }^{19,20} \mathrm{MoS}_{2}$, when thinned down to a ML, undergoes an indirect to direct bandgap transition, as illustrated in Figure 2. We have shown that this phenomenon is not restricted to $\mathrm{MoS}_{2}$ but is also present in other Group $6 \mathrm{MX}_{2}$ materials. ${ }^{21}$

Figure 2 also shows the importance of relativistic effects. Schwingenschlögl et al. ${ }^{39}$ predicted giant spin-orbit (SO) splitting for $\mathrm{MoS}_{2} \mathrm{ML}$ on the basis of density functional theory calculations. This strong quantum effect alters the electronic levels of the material, depending on their spin signature, due to the interaction of the intrinsic electron spin with its angular orbital momentum. This effect is also general for all $2 \mathrm{H}$ Group $6 \mathrm{MX}_{2}$ materials, with values reaching $480 \mathrm{meV}$ for $\mathrm{WTe}_{2} \cdot{ }^{40}$

It is intriguing to compare the electronic property changes of $\mathrm{MX}_{2}$ MLs and BLs. While MLs show giant SO splittings, these are absent in the bulk and BLs, which is explained by the presence of an inversion center, resulting in the presence of two degenerate orbitals of opposite spin for any point in the band structure. Thus, it is possible to exploit the symmetry for tuning the SO splitting, offering interesting materials for spintronic applications. Any symmetry breaking will introduce SO splitting in the BL. One way to do this is by an external electric field normal to the basal plane, for example, provided 
Table I. Overview of stable layered transition-metal dichalcogenides.

\begin{tabular}{|l|c|c|}
\hline Electrical Property & Material & Possible Role in Electronic Devices \\
\hline Metallic & $\mathrm{NbSe}_{2},{ }^{27} \mathrm{NbS}_{2}, \mathrm{NbTe}_{2}$ & $\begin{array}{c}\text { Interconnects, Josephson junctions, } \\
\text { superconducting qubits }\end{array}$ \\
\hline $\mathrm{TaS}_{2}, \mathrm{TaSe}_{2}, \mathrm{TaTe}_{2}$ & \\
\hline $\mathrm{ScS}_{2}, \mathrm{ScSe}_{2}, \mathrm{ScTe}_{2}$ & \\
\hline $\mathrm{TiS}_{2}, \mathrm{TiSe}_{2}, \mathrm{TiTe}_{2}$ & \\
\hline $\mathrm{VS}_{2}, \mathrm{VSe}_{2}, \mathrm{VTe}_{2}$ & \\
\hline $\mathrm{MnS}_{2}, \mathrm{MnSe}_{2}, \mathrm{MnTe}_{2}$ & \\
\hline $\mathrm{FeS}_{2}, \mathrm{FeSe}_{2}, \mathrm{FeTe}_{2}$ & \\
\hline Semimetallic & $\mathrm{NiTe}_{2}$ & \\
\hline Semiconducting & $\mathrm{TiSe}_{2}, \mathrm{WTe}_{2}{ }^{28}$ & Interconnects \\
\hline $\mathrm{MoS}_{2},{ }^{8} \mathrm{MoSe}_{2},{ }^{29} \mathrm{MoTe}_{2}$ & Transistors, switches, sensors, \\
optoelectronic devices
\end{tabular}

First experimental studies on mesoscopic structures have been performed on compounds in bold. $2 \mathrm{H}$ (trigonal prismatic) structure; most stable are in red. ${ }^{25}$

by a gate voltage, as shown in Figure 3. This introduces a Stark effect - the applied electric field quasi-linearly splits the bands - and the SO splitting converges quickly to about the same values as found for the respective MLs. ${ }^{41}$ After a field strength of $\sim 5 \mathrm{~V} / \mathrm{nm}$, a value that can be reached experimentally, the SO splitting is saturated. However, $\mathrm{MX}_{2} \mathrm{MLs}$ are insensitive to the presence of even strong gate voltages. ${ }^{42}$

External fields have another important effect on the electronic structure of $\mathrm{MX}_{2}$ materials and can be used to tune the bandgap. Again, MLs are almost unaffected by external electric fields, with bandgap and effective electron and hole masses remaining stable. ${ }^{42}$ Only at very high fields of about $50 \mathrm{~V} / \mathrm{nm}$ do the bandgaps get smaller and eventually vanish, but at such a high field strength in the laboratory, MLs would not remain intact. BLs show a quasi-linear decrease in the bandgap in the presence of an external field (Figure 4).

Mechanical deformation is another way to change the electronic structure in $\mathrm{MX}_{2}$ materials. Group $62 \mathrm{H} \mathrm{MX}$ materials readily form nanotubes and nanoonions, which have been investigated for their tribological properties. $\mathrm{WS}_{2}$ nanotubes resist strain for elongations of $10 \%$ and more, thereby showing a linear strainstress relation up to rupture. ${ }^{43}$ In the 2D crystal, deformation is accompanied by strong electronic effects (straintronics). Under isotropic and unidirectional strain, the bandgap is almost linearly lowered until a semiconductormetal transition is observed at about $10 \%$ isotropic strain (Figure 4). ${ }^{44}$ Note that strained $\mathrm{MoS}_{2} \mathrm{ML}$, as well as related Group $6 \mathrm{MX}_{2} \mathrm{MLs}$, are no longer direct bandgap semiconductors.

The metallic state has been confirmed by explicit calculations of the quantum conductance, indicating that transport

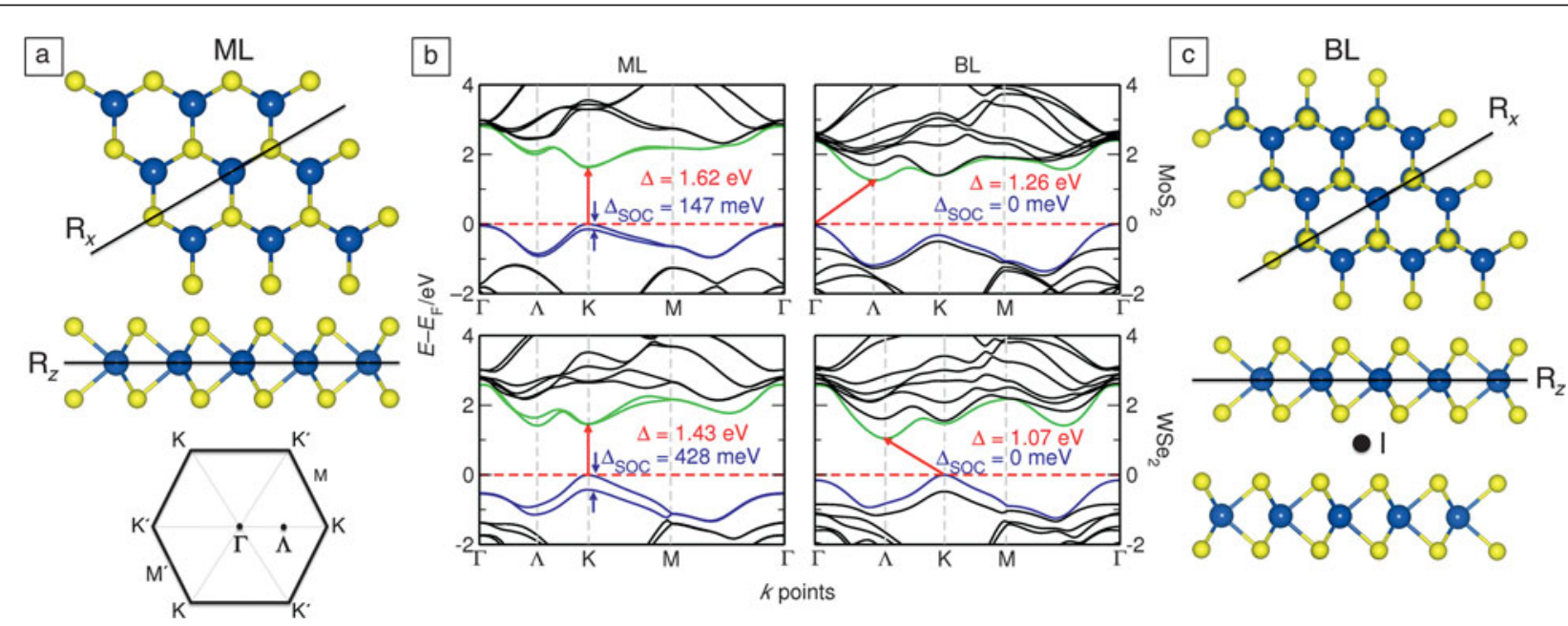

Figure 2. (a) Structure of $2 \mathrm{H} \mathrm{MX}$ monolayers (MLs) with high-symmetry reflection planes $\mathrm{R}_{x}$ and $\mathrm{R}_{z}$, and the Brillouin zone with its highsymmetry $k$ points. (c) The same information for the bilayer (BL) system, with the inversion point I indicated. (b) Band structure of MoS ${ }_{2}$ (top) and $\mathrm{WSe}_{2}$ (bottom) for $\mathrm{ML}(\mathrm{a})$ and $\mathrm{BL}$ (c). The Fermi level $\left(E_{\mathrm{F}}\right)$ is indicated by a red dashed line. The valence band (blue) and conduction band (green) frontier orbitals are marked. The red arrow shows the lowest energy electronic excitation, indicating a direct bandgap for the MLs, but an indirect one for the BLs. Bandgaps $(\Delta)$ and spin-orbit splitting $\left(\Delta_{\mathrm{soc}}\right)$ are indicated. Adapted with permission from Reference 40. 


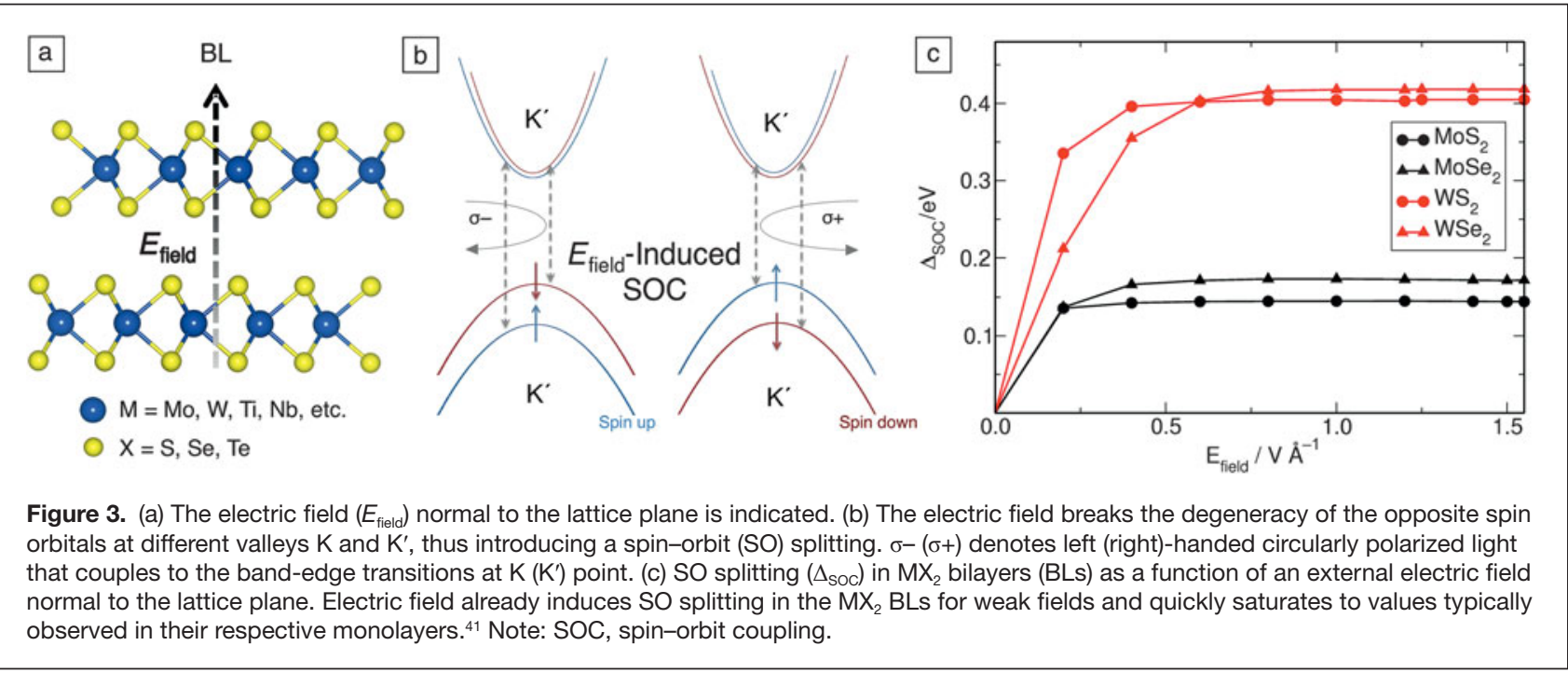

channels are opened. Unidirectional strain gives similar results but with a smaller magnitude. Also, unidirectional strain can be employed to introduce anisotropy to the electronic properties. ${ }^{45}$ However, it is difficult to introduce strain experimentally. One way is to employ an atomic force microscope (AFM) tip; computer simulations of an indentation experiment showed that the electronic structure of $\mathrm{MoS}_{2}$ remains remarkably stable up to the point of piercing by the tip. ${ }^{46}$ Another method is bending, or thermal expansion, which could be carried out on a substrate with a strong thermal expansion coefficient. However, due to the low friction coefficient of Group $6 \mathrm{MX}_{2}$, only a small amount of isotropic strain or compression can be introduced into a $\mathrm{MoS}_{2} \mathrm{ML} .{ }^{47}$ Nevertheless, the first experiments with strain up to $2 \%$ confirm that the bandgap of $\mathrm{MoS}_{2}$ ML can be tuned by strain with an accompanying bandgap decrease of $\sim 70 \mathrm{meV}$ per $\%$ strain. $^{48,49}$
The electronic properties of $\mathrm{MX}_{2}$ materials are strongly affected by defects that often form due to the synthesis procedure. For instance, point defects lead to new photoemission peaks and enhanced photoluminescence intensity in $\mathrm{MoS}_{2}$ MLs. ${ }^{50}$ These effects can be attributed to the trapping potential of free charge carriers and to localized excitons. Simulations of the electronic structure of defective $\mathrm{MoS}_{2} \mathrm{ML}$ show that defects introduce mid-gap states that act as scattering centers. ${ }^{51}$ Our non-equilibrium Green's function transport simulations, where the current due to a potential drop between two leads in the presence of a bias voltage is calculated, show that single atomic vacancies can significantly reduce the average conductance and lead to significant anisotropy of electron transfer in MLs with grain boundaries. These results indicate that defects are important limiting factors in electronic transport, ${ }^{52}$ thus explaining a large variation of conductivity in different samples.

Similar to freestanding graphene, $\mathrm{MX}_{2}$ MLs are not completely flat, which was shown from the microscopic structure of thin $\mathrm{MoS}_{2}$ layers using high-resolution transmission electron microscopy and atomic force microscopy. ${ }^{53} \mathrm{MoS}_{2}$ MLs form ripples, which may reach heights of 6-10 $\AA$ for a lateral flake length of $6-10 \mathrm{~nm}$. This was supported by Born-Oppenheimer molecular dynamics simulations. Here, forces on the atoms are calculated from first principles, while the dynamics of the atoms is calculated using classical theory. These simulations confirmed that the inherent dynamics of $\mathrm{MoS}_{2}$ MLs lead to spontaneous ripple formation even at temperatures as low as $77 \mathrm{~K} .{ }^{54}$ The ripples converged to the heights observed experimentally for supercells with lateral lengths of about $9 \mathrm{~nm}$. 


\section{Field-effect devices}

One of the most direct ways of accessing the electrical properties of a material is to incorporate it in an electronic device, for example in a FET (see Figure 5). In this multi-terminal device, current is injected by applying a voltage between source and drain electrodes, while the charge density can be modulated and the Fermi level varied using the gate electrode. FETs are not just convenient devices for probing the electrical properties of materials, but they are also the main building blocks of modern electronics, so demonstrating that a well-behaved FET can be made using a new semiconducting material is an important step in highlighting their technological relevance.

Radisavljevic et al. reported the first FET based on a 2D TMDC material in 2011..$^{7}$ It included a $\mathrm{HfO}_{2}$ top-gate dielectric and showed a current ON/OFF ratio exceeding $10^{8}$ at room temperature and OFF-state currents smaller than $25 \mathrm{fA} / \mu \mathrm{m}$. For comparison, an ON/OFF ratio of $10^{6}$ and an OFF-state current on the order of $1 \mathrm{nA} / \mu \mathrm{m}$ is considered acceptable for digitial electronic circuits. This showed for the first time that electronic devices with interesting performance levels could be realized based on 2D materials other than graphene. It resulted in the inclusion of $\mathrm{MoS}_{2}$ and semiconducting TMDCs in the International Technology Roadmap for Semiconductors ${ }^{55}$ in 2011.

The encapsulation of $\mathrm{MoS}_{2}$ using insulating $\mathrm{HfO}_{2}$ was crucial in achieving high performance levels in early devices. Subsequent temperature-dependent measurements of electrical transport in various geometries ${ }^{56}$ showed that encapsulation in $\mathrm{HfO}_{2}$ can effectively increase the mobility of $\mathrm{MoS}_{2}$ through a combination of homopolar phonon mode quenching ${ }^{57,58}$ as well as charged impurity screening by the dielectric and the top gate. ${ }^{59}$ A complementary approach to achieving high mobility involves vacuum annealing, resulting in the removal of adsorbates, ${ }^{60,61}$ lower noise, ${ }^{62}$ and low-temperature mobilities in $\mathrm{ML} \mathrm{MoS}_{2}$ of $\sim 250 \mathrm{~cm}^{2} / \mathrm{Vs}$ extracted from Hall effect measurements and field-effect effective mobilities of $\sim 1000 \mathrm{~cm}^{2} / \mathrm{Vs}^{61}$ These values could be significantly underestimated and lower than the intrinsic limit ${ }^{57}$ due to the presence of electronic trap states ${ }^{63}$ and the formation of a band tail, as seen in Figure 6a. The smearing of the band edge due to various types of inhomogeneities could include sulfur vacancies, charges trapped at the TMDC/dielectric interface, or other structural defects. The extent of the band tail in $\mathrm{MoS}_{2}$ was characterized by Zhu et al., who found a $\sim 100 \mathrm{meV}$ characteristic energy width of the band tail using frequency-dependent capacitance-voltage measurements ${ }^{63}$ (Figure 6b).

While further increases in the mobility of $\mathrm{MoS}_{2}$ are to be expected, it is already large enough to allow operation of $\mathrm{MoS}_{2}$-based transistors in the GHz range, where current, voltage, and power gains were demonstrated ${ }^{64}$ These performance figures are mostly limited by the contact resistance.

Reducing the contact resistance due to the Schottky barrier between the $2 \mathrm{D}$ semiconductor and the metal and realizing an ohmic contact is one of the most important challenges for engineering a high-quality device. This is, for example, crucial in achieving highly responsive photodetectors. ${ }^{65}$ One way to achieve this is by using low work-function metals as contacts, such as $\mathrm{Ni}^{66}$ or Sc. ${ }^{67,68}$ In addition to choosing the metal with an advantageous work function, other factors such as the orbital overlap between the metals and TMDCs and the contact geometry (top versus edge contacts) also play important roles, as shown by Kang et al. first in their theoretical study ${ }^{69}$ and later in the experimental demonstration of low-resistance Mo contacts to $\mathrm{MoS}_{2}{ }^{70}$

A complementary approach is to reduce the Schottky barrier height by doping the semiconducting region under the contact (e.g., by $p$-doping $\mathrm{WSe}_{2}$ devices to $\mathrm{NO}_{2}{ }^{31}$ in order to improve Pd contacts, $n$-type doping using the chemical compound benzyl viologen to improve Ni contacts to $\mathrm{MoS}_{2},{ }^{71}$ or chlorine doping in the case of $\mathrm{Ni}$ on $\mathrm{WS}_{2}$ and $\left.\mathrm{MoS}_{2}\right){ }^{72}$
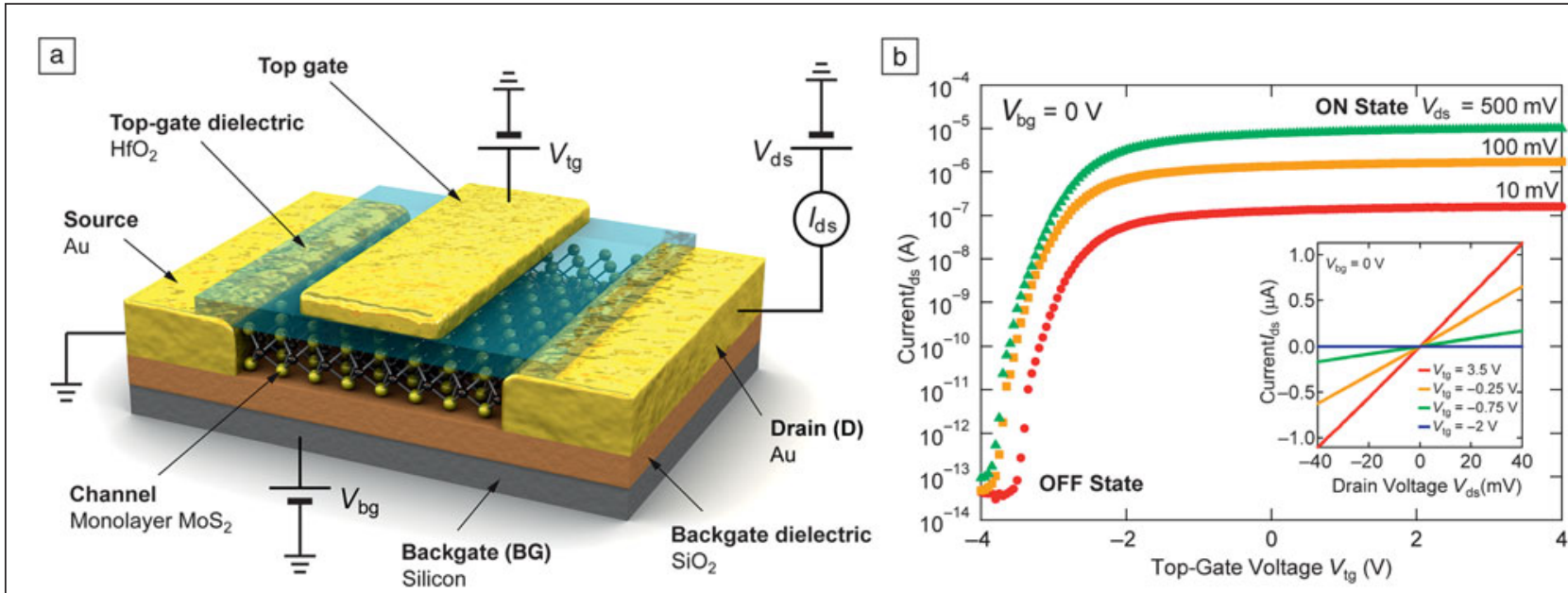

Figure 5. (a) Schematic representation of the first top-gated field-effect transistor with a 2D transition-metal dichalcogenide (MoS ${ }_{2}$ ) channel. (b) Device current as a function of gate voltage. The transistor could be completely turned OFF for gate voltages <-2 V, and it showed an ON/OFF current ratio of $10^{8}$. Inset: current as a function of bias voltage, showing linear $I_{\mathrm{ds}}-V_{\mathrm{ds}}$ curves for small bias voltages. ${ }^{7}$ 

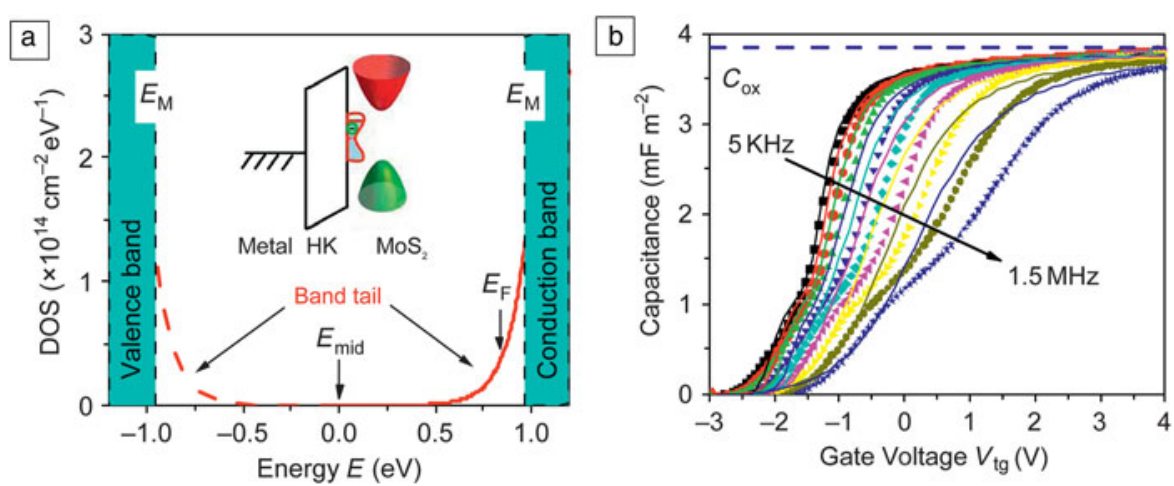

that, in principle, all parts of an FET structure can be replaced by $2 \mathrm{D}$ materials. The lack of dangling bonds in 2D materials also weakens the usual constraints of lattice matching that limit the possible combinations for "classical" three-dimensional materials. This allows for the realization of a host of new types of devices, such as vertical FETs with several $\mathrm{BN}^{12}$ or TMDC layers $^{79}$ sandwiched between graphene electrodes.

Being a good conductor, graphene

Figure 6. (a) Density of states (DOS) for $\mathrm{MoS}_{2}$, including both localized band-tail and mid-gap localized states. $E_{\text {mid }}$ is the energy level in the middle of the bandgap and $E_{M}$ is the energy of the mobility edge. Inset shows a simplified band diagram of the device. (b) Multi-frequency capacitance of a $\mathrm{MoS}_{2}$ test structure as a function of gate voltage $\left(V_{\mathrm{tg}}\right) . C_{\mathrm{ox}}$ is the oxide capacitance. The symbols are experimental results, and the lines indicate modeling results. ${ }^{63}$ Data and the model show reasonable agreement. Adapted with permission from Reference 63. () 2014 Nature Publishing Group. Note: HK, high-к (dielectric constant) dielectric; $E_{\mathrm{F}}$, Fermi energy.

The biggest reduction in contact resistance reported so far was by using the radical approach of converting $\mathrm{MoS}_{2}$ in the contact region from the semiconducting $2 \mathrm{H}$ to the metallic $1 \mathrm{~T}$ phase by locally increasing the charge doping level using lithiation, ${ }^{73}$ resulting in a contact resistance of less than $300 \Omega \cdot \mu \mathrm{m}$. Previous typical contact resistance values were above $1 \mathrm{k} \Omega \cdot \mu \mathrm{m}$. Not only can $\mathrm{MoS}_{2}$ be semiconducting or metallic, but at sufficiently high doping levels, it can also become superconducting, with a gate tunable transition temperature showing a maximum at $11 \mathrm{~K} .{ }^{74}$

Because 2D TMDCs are direct bandgap semiconductors, FETs based on these materials can also be used as simple and efficient photodetectors. Early phototransistors based on $\mathrm{MoS}_{2}$ had a photoresponsivity of $7.5 \mathrm{~mA} / \mathrm{W} .{ }^{75}$ Using layers with different thicknesses, the responsivity of the photodetector can be tuned to different wavelength ranges. ${ }^{76}$ Lopez-Sanchez et al. ${ }^{65}$ reported $\mathrm{ML} \mathrm{MoS}_{2}$ phototransistors with a high photoresponsivity of $880 \mathrm{~A} / \mathrm{W}$.

Experimental work on other semiconducting TMDCs has also started. While $\mathrm{MoS}_{2}$ is usually $n$-type, $p$-type FET devices based on $\mathrm{WSe}_{2}$ have been realized showing a roomtemperature hole mobility of $250 \mathrm{~cm}^{2} / \mathrm{Vs}^{31}$ as well as $n$ - $\mathrm{WSe}_{2}$ transistors with a roomtemperature mobility of $142 \mathrm{~cm}^{2} / \mathrm{Vs}^{32}$ Using liquid gating, in which an ionic liquid is used in place of a solid dielectric, it was possible to achieve ambipolar operation in $\mathrm{ML} \mathrm{WSe}_{2}{ }^{77}$ as well as in $\mathrm{WS}_{2}{ }^{78}$

While most of the work in 2D TMDC-based transistors has focused on using a $2 \mathrm{D}$ material as the semiconducting channel, the wide range of electrical properties represented by $2 \mathrm{D}$ materials, from insulating such as boron nitride $(\mathrm{BN})$ to superconducting, implies

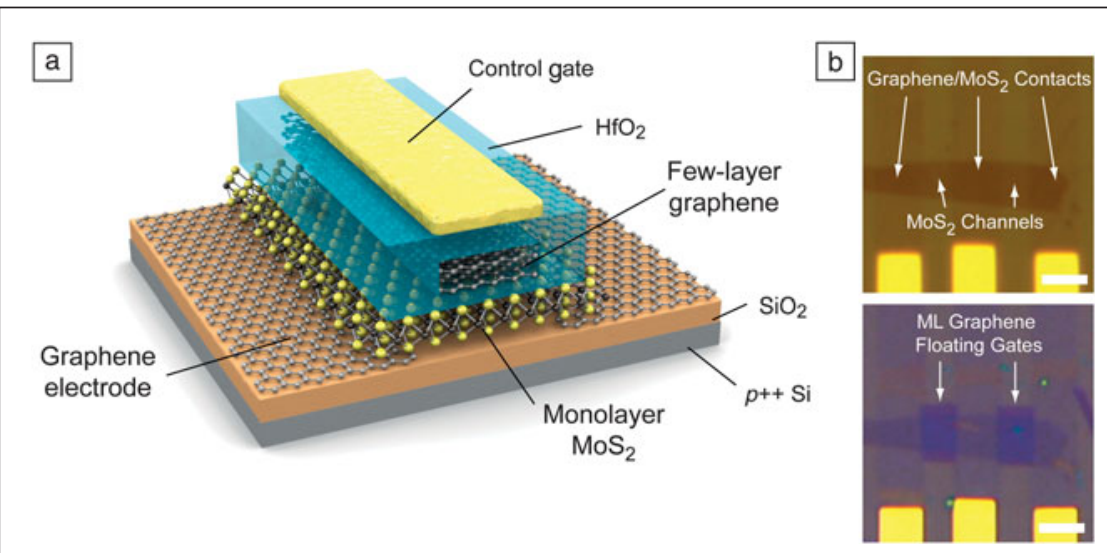

Figure 7. (a) $\mathrm{MoS}_{2}$ /graphene heterostructure memory based on a $\mathrm{MoS}_{2}$ transistor with graphene contacts. (b) Optical image of $\mathrm{MoS}_{2}$ contacted by graphene (upper panel) and multilayer graphene floating gates deposited on top of the semiconducting channel (lower panel). ${ }^{80}$ Note: ML, monolayer. 
the ones used in consumer electronic devices such as cell phones and cameras, in a heterostructure geometry. Such devices could offer reduced crosstalk between neighboring memory cells and could be scaled to smaller sizes due to the high sensitivity of the $\mathrm{ML} \mathrm{MoS}$ semiconducting channel to the charge stored on the multilayer graphene floating gate.

\section{Flexible devices}

In addition to its interesting electronic applications, TMDC semiconductors have favorable mechanical properties. The first mechanical measurements of the stiffness and breaking strength of single and $\mathrm{BL} \mathrm{MoS}$, were carried out by suspending $\mathrm{MoS}_{2}$ membranes on perforated substrates and deforming them using an AFM tip. ${ }^{86}$ These experiments showed that $\mathrm{MoS}_{2}$ has a Young's modulus of $\sim 270 \mathrm{GPa}$, higher than that of steel ( $205 \mathrm{GPa})$. The breaking strength of single and BL $\mathrm{MoS}_{2}$ was between $6 \%$ and $11 \%$ of their respective Young's modulus, which represents the upper theoretical limit of a material's breaking strength ${ }^{87}$ and reflects the intrinsic properties of its interatomic bonds. This makes $\mathrm{ML} \mathrm{MoS}_{2}$ the strongest semiconducting material and suggests that $\mathrm{MoS}_{2}$ is suitable for integration with flexible plastic substrates, such as polyimide, whose maximum strain before failure is $\sim 7 \%$. Similar mechanical properties are expected in all TMDC materials because of the similar nature of interatomic bonds in these materials. Therefore, $\mathrm{MoS}_{2}$ is an interesting material for flexible electronics and nanoelectromechanical systems. CastellanosGomez et al ${ }^{88}$ realized the first devices of this kind when they demonstrated nanomechanical resonators based on $\mathrm{ML} \mathrm{MoS}_{2}$ membranes with resonant frequencies of up to $30 \mathrm{MHz}$. A number of flexible FETs based on $\mathrm{MoS}_{2}$ have been realized using liquid gels, ${ }^{89}$ high- $\kappa$ (where $\kappa$ is the dielectric constant), ${ }^{90}$ or $\mathrm{BN}^{91}$ as dielectrics, and show robust electrical operation against mechanical deformation.

\section{Summary}

We have summarized the most recent work on $\mathrm{MoS}_{2}$ and other Group 6 TMDC materials, focusing on their electronic properties and their potential to enter the field of next-generation 2D electronics, including applications in low-power electronics (due to reduction of leakage currents and contact resistance), flexible electronics, optoelectronics, and straintronics. The enormous progress of the field, ranging from fundamental science up to device implementation, is reflected by the large number of scientists working in the field, even though the first transistor on a $\mathrm{MoS}_{2} \mathrm{ML}$ was reported only recently. $\mathrm{MoS}_{2}$ and other TMDCs are being developed, in synergy with graphene and hexagonal boron nitride, as important material components for 2D electronics. The work has just begun, and a large number of TMDCs have not even been synthesized or studied.

\section{Acknowledgments}

This work was financially supported by the European Research Council (grants no. 240076 \& no. 256962), Marie
Curie ITN network "MoWSeS" (grant no. 317451), the Swiss National Science Foundation (grants no. 132102 and 138237), Swiss SNF Sinergia Grant no. 147607, and Deutsche Forschungsgemeinschaft (HE 3543/19-1).

\section{References}

1. A. Kuc, T. Heine, Chem. Soc. Rev. (2014), doi, 10.1039/C4CS00276H.

2. Q.H. Wang, K. Kalantar-Zadeh, A. Kis, J.N. Coleman, M.S. Strano, Nat. Nanotechnol. 7, 699 (2012).

3. K.S. Novoselov, A.K. Geim, S.V. Morozov, D. Jiang, Y. Zhang, S.V. Dubonos, I.V. Grigorieva, A.A. Firsov, Science 306, 666 (2004).

4. K.S. Novoselov, Rev. Mod. Phys. 83, 837 (2011).

5. Y. Zhang, T.-T. Tang, C. Girit, Z. Hao, M.C. Martin, A. Zettl, M.F. Crommie, Y.R. Shen, F. Wang, Nature 459, 820 (2009).

6. F. Xia, D.B. Farmer, Y. Lin, P. Avouris, Nano Lett. 10, 715 (2010).

7. B. Radisavljevic, A. Radenovic, J. Brivio, V. Giacometti, A. Kis, Nat. Nanotechnol.

6, 147 (2011)

8. F. Wypych, R. Schollhorn, J. Chem. Soc. Chem. Commun. 19, 1386 (1992).

9. K.S. Novoselov, D. Jiang, F. Schedin, T.J. Booth, V.V. Khotkevich, S.V. Morozov, A.K. Geim, Proc. Natl. Acad. Sci. U.S.A. 102, 10451 (2005).

10. M.M. Benameur, B. Radisavljevic, J.S. Heron, S. Sahoo, H. Berger, A. Kis, Nanotechnology 22, 125706 (2011).

11. J.N. Coleman, M. Lotya, A. O'Neill, S.D. Bergin, P.J. King, U. Khan, K. Young, A. Gaucher, S. De, R.J. Smith, I.V. Shvets, S.K. Arora, G. Stanton, H.Y. Kim, K. Lee, G.T. Kim, G.S. Duesberg, T. Hallam, J.J. Boland, J.J. Wang, J.F. Donegan, J.C. Grunlan, G. Moriarty, A. Shmeliov, R.J. Nicholls, J.M. Perkins, E.M. Grieveson, K. Theuwissen, D.W. McComb, P.D. Nellist, V. Nicolosi, Science 331, 568 (2011).

12. S. Najmaei, Z. Liu, W. Zhou, X. Zou, G. Shi, S. Lei, B.I. Yakobson, J.-C. Idrobo, P.M. Ajayan, J. Lou, Nat. Mater. 12, 754 (2013)

13. A.M. van der Zande, P.Y. Huang, D.A. Chenet, T.C. Berkelbach, Y. You, G.-H. Lee, T.F. Heinz, D.R. Reichman, D.A. Muller, J.C. Hone, Nat. Mater. 12, $554(2013)$

14. L. Britnell, R.V. Gorbachev, R. Jalil, B.D. Belle, F. Schedin, A. Mishchenko, T. Georgiou, M.I. Katsnelson, L. Eaves, S.V. Morozov, N.M.R. Peres, J. Leist, A.K. Geim, K.S. Novoselov, L.A. Ponomarenko, Science 335, 947 (2012).

15. J.A. Wilson, A.D. Yoffe, Adv. Phys. 18, 193 (1969).

16. R.F. Frindt, A.D. Yoffe, Proc. R. Soc. Lond. A 273, 69 (1963)

17. P. Joensen, R.F. Frindt, S.R. Morrison, Mater. Res. Bull. 21, 457 (1986).

18. K.K. Kam, B.A. Parkinson, J. Phys. Chem. 86, 463 (1982).

19. A. Splendiani, L. Sun, Y. Zhang, T. Li, J. Kim, C.-Y. Chim, G. Galli, F. Wang, Nano Lett. 10, 1271 (2010).

20. K.F. Mak, C. Lee, J. Hone, J. Shan, T.F. Heinz, Phys. Rev. Lett. 105, 136805 (2010).

21. A. Kuc, N. Zibouche, T. Heine, Phys. Rev. B Condens. Matter 83, 245213 (2011). 22. T. Li, G. Galli, J. Phys. Chem. C111, 16192 (2007).

23. L. Liu, S.B. Kumar, Y. Ouyang, J. Guo, IEEE Trans. Electron Devices 58 , 3042 (2011).

24. Y. Ding, Y. Wang, J. Ni, L. Shi, S. Shi, W. Tang, Physica B 406, 2254 (2011).

25. C. Ataca, H. Şahin, S. Ciraci, J. Phys. Chem. C116, 8983 (2012).

26. Acc. Chem. Res. 48, 1 (2015).

27. N.E. Staley, J. Wu, P. Eklund, Y. Liu, L. Li, Z. Xu, Phys. Rev. B Condens. Matter 80, 184505 (2009).

28. M.N. Ali, J. Xiong, S. Flynn, J. Tao, Q.D. Gibson, L.M. Schoop, T. Liang, N. Haldolaarachchige, M. Hirschberger, N.P. Ong, R.J. Cava, Nature 514, 205 (2014).

29. S. Tongay, J. Zhou, C. Ataca, K. Lo, T.S. Matthews, J. Li, J.C. Grossman, J. Wu, Nano Lett. 12, 5576 (2012).

30. W.S. Hwang, M. Remskar, R. Yan, V. Protasenko, K. Tahy, S.D. Chae, P. Zhao, A. Konar, H. Xing, A. Seabaugh, D. Jena, Appl. Phys. Lett. 101, 013107 (2012).

31. H. Fang, S. Chuang, T.C. Chang, K. Takei, T. Takahashi, A. Javey, Nano Lett. 12, 3788 (2012).

32. W. Liu, J. Kang, D. Sarkar, Y. Khatami, D. Jena, K. Banerjee, Nano Lett. 13, 1983 (2013).

33. P. Miró, M. Audiffred, T. Heine, Chem. Soc. Rev. 43, 6537 (2014).

34. S. Tongay, H. Sahin, C. Ko, A. Luce, W. Fan, K. Liu, J. Zhou, Y.-S. Huang, C.-H. Ho, J. Yan, D.F. Ogletree, S. Aloni, J. Ji, S. Li, J. Li, F.M. Peeters, J. Wu., Nat. Commun. 5, 3252 (2014).

35. S. Yang, S. Tongay, Q. Yue, Y. Li, B. Li, F. Lu, Sci. Rep. 4, 5442 (2014).

36. T. Heine, Acc. Chem. Res. 48, 65 (2015).

37. P. Miro, M. Audiffred, T. Heine, Chem. Soc. Rev. 43, 6537 (2014).

38. P. Miro, M. Ghorbani-Asl, T. Heine, Angew. Chem. Int. Ed. 53, 3015 (2014).

39. Z.Y. Zhu, Y.C. Cheng, U. Schwingenschlögl, Phys. Rev. B Condens. Matter 84, 153402 (2011)

40. N. Zibouche, A. Kuc, J. Musfeldt, T. Heine, Ann. Phys. 526, 395 (2014). 
41. N. Zibouche, P. Philipsen, A. Kuc, T. Heine, Phys. Rev. B Condens. Matter 90, 125440 (2014).

42. N. Zibouche, P. Philipsen, T. Heine, A. Kuc, Phys. Chem. Chem. Phys. 16, 11251 (2014).

43. I. Kaplan-Ashiri, S.R. Cohen, K. Gartsman, V. Ivanovskaya, T. Heine, G. Seifert, I. Wiesel, H.D. Wagner, R. Tenne, Proc. Natl. Acad. Sci. U.S.A. 103, 523 (2006). 44. M. Ghorbani-Asl, S. Borini, A. Kuc, T. Heine, Phys. Rev. B Condens. Matter 87, 235434 (2013).

45. M. Ghorbani-AsI, N. Zibouche, M. Wahiduzzaman, A.F. Oliveira, A. Kuc, T. Heine, Sci. Rep. 3, 2961, (2013).

46. T. Lorenz, M. Ghorbani-Asl, J.-0. Joswig, T. Heine, G. Seifert, Nanotechnology 25, 445201 (2014).

47. G. Plechinger, A. Castellanos-Gomez, M. Buscema, H. van der Zant, G. Steele, A. Kuc, T. Heine, C. Schüller, T. Korn, 2D Mater. (2015) (forthcoming). 48. K. He, C. Poole, K.F. Mak, J. Shan, Nano Lett. 13, 2931 (2013).

49. H.J. Conley, B. Wang, J.I. Ziegler, R.F. Haglund, S.T. Pantelides, K.I. Bolotin, Nano Lett. 13, 3626 (2013).

50. S. Tongay, J. Suh, C. Ataca, W. Fan, A. Luce, J.S. Kang, J. Liu, C. Ko, R. Raghunathanan, J. Zhou, F. Ogletree, J. Li, J.C. Grossman, J. Wu, Sci. Rep. 3 , 2657, (2013)

51. M. Ghorbani-Asl, A.N. Enyashin, A. Kuc, G. Seifert, T. Heine, Phys. Rev. B Condens. Matter 88, 245440 (2013).

52. H. Qiu, T. Xu, Z. Wang, W. Ren, H. Nan, Z. Ni, Q. Chen, S. Yuan, F. Miao, F. Song, G. Long, Y. Shi, L. Sun, J. Wang, X. Wang, Nat. Commun. 4, 2642, (2013)

53. J. Brivio, D.T.L. Alexander, A. Kis, Nano Lett. 11, 5148 (2011).

54. P. Miro, M. Ghorbani-Asl, T. Heine, Adv. Mater. 25, 5473 (2013).

55. International Technology Roadmap for Semiconductors (2011), http://www. itrs.net/.

56. B. Radisavljevic, A. Kis, Nat. Mater. 12, 815 (2013).

57. K. Kaasbjerg, K.S. Thygesen, K.W. Jacobsen, Phys. Rev. B Condens. Matter 85, 115317 (2012)

58. K. Kaasbjerg, K.S. Thygesen, A.-P. Jauho, Phys. Rev. B Condens. Matter 87. $235312(2013)$

59. Z.-Y. Ong, M.V. Fischetti, Phys. Rev. B Condens. Matter 88, 165316 (2013). 60. D. Jariwala, V.K. Sangwan, D.J. Late, J.E. Johns, V.P. Dravid, T.J. Marks, L.J. Lauhon, M.C. Hersam, Appl. Phys. Lett. 102, 173107 (2013).

61. B. Baugher, H.O.H. Churchill, Y. Yang, P. Jarillo-Herrero, Nano Lett. 13, 4212 (2013).

62. X. Xie, D. Sarkar, W. Liu, J. Kang, O. Marinov, M.J. Deen, K. Banerjee, ACS Nano 8, 5633 (2014).

63. W. Zhu, T. Low, Y.-H. Lee, H. Wang, D.B. Farmer, J. Kong, F. Xia, P. Avouris, Nat. Commun. 5, 3087 (2014).

64. D. Krasnozhon, D. Lembke, C. Nyffeler, Y. Leblebici, A. Kis, Nano Lett. 14, 5905 (2014)

65. O. Lopez-Sanchez, D. Lembke, M. Kayci, A. Radenovic, A. Kis, Nat. Nanotechnol. 8, 497 (2013).
66. A.T. Neal, H. Liu, J.J. Gu, P.D. Ye, Proc. 2012 70th Ann. Dev. Res. Conf. (DRC) (2012), p. 65

67. I. Popov, G. Seifert, D. Tománek, Phys. Rev. Lett. 108, 156802 (2012).

68. S. Das, H.-Y. Chen, A.V. Penumatcha, J. Appenzeller, Nano Lett. 13, 100 (2013) 69. J. Kang, W. Liu, D. Sarkar, D. Jena, K. Banerjee, Phys. Rev. X 4, 031005 (2014)

70. J. Kang, W. Liu, K. Banerjee, Appl. Phys. Lett. 104, 233502 (2014).

71. D. Kiriya, M. Tosun, P. Zhao, J.S. Kang, A. Javey, J. Am. Chem. Soc. $\mathbf{1 3 6}$ 7853 (2014)

72. L. Yang, K. Majumdar, H. Liu, Y. Du, H. Wu, M. Hatzistergos, P.Y. Hung, R. Tieckelmann, W. Tsai, C. Hobbs, P.D. Ye, Nano Lett. (2014), available at http://arxiv.org/pdf/1410.8201

73. R. Kappera, D. Voiry, S.E. Yalcin, B. Branch, G. Gupta, A.D. Mohite, M. Chhowalla, Nat. Mater. 13, 1128 (2014).

74. J.T. Ye, Y.J. Zhang, R. Akashi, M.S. Bahramy, R. Arita, Y. Iwasa, Science 338 1193 (2012)

75. Z. Yin, H. Li, H. Li, L. Jiang, Y. Shi, Y. Sun, G. Lu, Q. Zhang, X. Chen, H. Zhang, ACS Nano 6, 74 (2012).

76. H.S. Lee, S.-W. Min, Y.-G. Chang, M.K. Park, T. Nam, H. Kim, J.H. Kim, S. Ryu, S. Im, Nano Lett. 12, 3695 (2012)

77. A. Allain, A. Kis, ACS Nano 8, 7180 (2014)

78. S. Jo, N. Ubrig, H. Berger, A.B. Kuzmenko, A.F. Morpurgo, Nano Lett. 14, 2019 (2014)

79. T. Georgiou, R. Jalil, B.D. Belle, L. Britnell, R.V. Gorbachev, S.V. Morozov, Y.-J. Kim, A. Gholinia, S.J. Haigh, O. Makarovsky, L. Eaves, L.A. Ponomarenko, A.K. Geim, K.S. Novoselov, A. Mishchenko, Nat. Nanotechnol. 8, 100 (2013).

80. S. Bertolazzi, D. Krasnozhon, A. Kis, ACS Nano 7, 3246 (2013).

81. T. Roy, M. Tosun, J.S. Kang, A.B. Sachid, S.B. Desai, M. Hettick, C.C. Hu, A. Javey, ACS Nano 8, 6259 (2014).

82. B. Radisavljevic, M.B. Whitwick, A. Kis, ACS Nano 5, 9934 (2011).

83. B. Radisavljevic, M.B. Whitwick, A. Kis, Appl. Phys. Lett. 101, 043103 (2012)

84. H. Wang, L. Yu, Y.-H. Lee, Y. Shi, A. Hsu, M.L. Chin, L.-J. Li, M. Dubey, J. Kong, T. Palacios, Nano Lett. 12, 4674 (2012).

85. M. Tosun, S. Chuang, H. Fang, A.B. Sachid, M. Hettick, Y. Lin, Y. Zeng, A. Javey, ACS Nano 8, 4948 (2014).

86. S. Bertolazzi, J. Brivio, A. Kis, ACS Nano 5, 9703 (2011).

87. A. Griffith, Philos. Trans. R. Soc Lond. A 221, 163 (1920)

88. A. Castellanos-Gomez, R. van Leeuwen, M. Buscema, H.S.J. van der Zant, G.A. Steele, W.J. Venstra, Adv. Mater. 25, 6719 (2013)

89. J. Pu, Y. Yomogida, K.-K. Liu, L.-J. Li, Y. Iwasa, T. Takenobu, Nano Lett. 12, 4013 (2012)

90. H.-Y. Chang, S. Yang, J. Lee, L. Tao, W.-S. Hwang, D. Jena, N. Lu, D. Akinwande, ACS Nano 7, 5446 (2013).

91. G.-H. Lee Y.-J. Yu, X. Cui, N. Petrone, C.-H. Lee, M.S. Choi, D.-Y. Lee, C. Lee, W.J. Yoo, K. Watanabe, T. Taniguchi, C. Nuckolls, P. Kim, J. Hone, ACS Nano 7 , 7931 (2013)

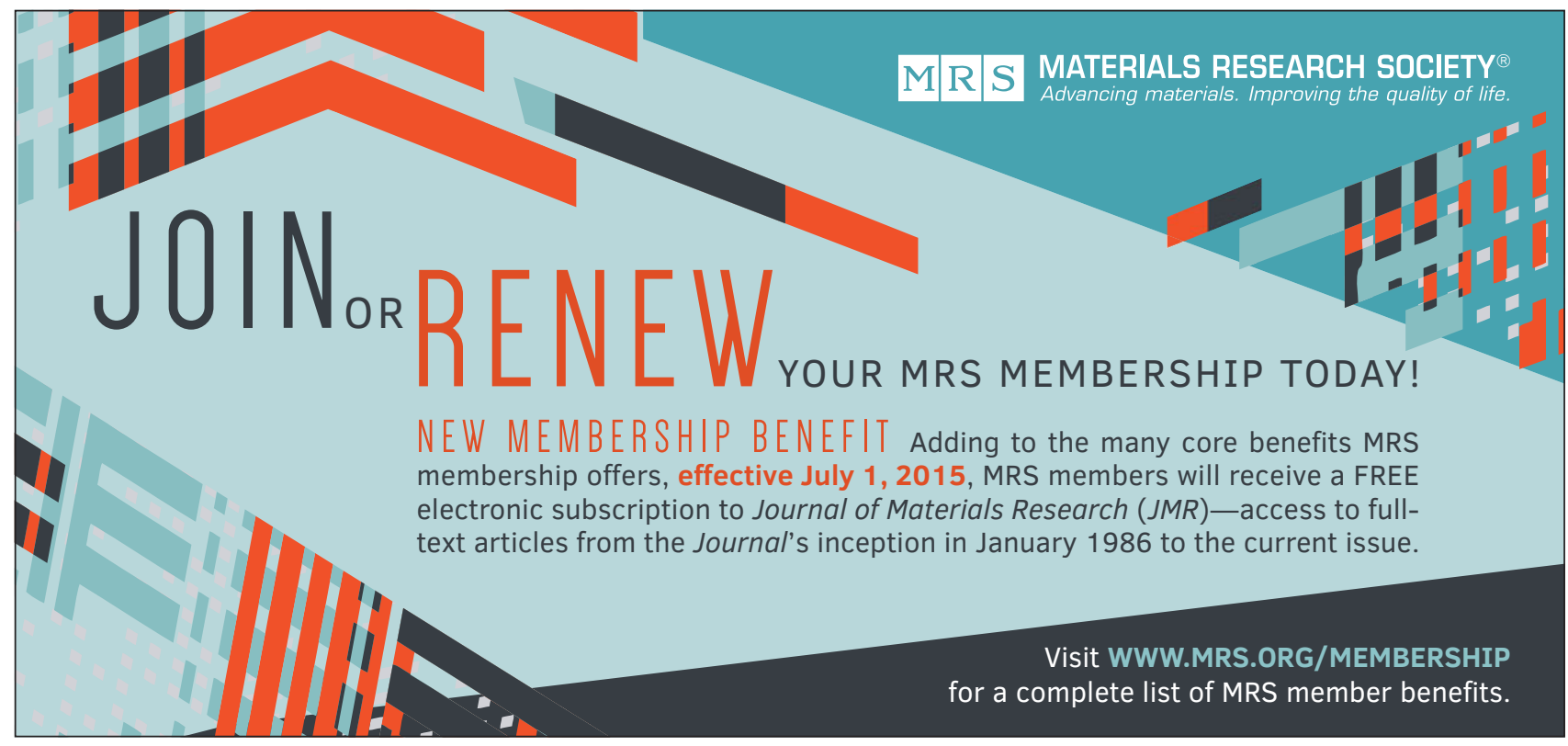

